\title{
Existence of a positive solution for quasilinear elliptic equations with nonlinearity including the gradient
}

\section{Mieko Tanaka*}

\section{"Correspondence:}

tanaka@ma.kagu.tus.ac.jp

Department of Mathematics, Tokyo

University of Science,

Kagurazaka 1-3, Shinjyuku-ku, Tokyo

162-8601, Japan

\begin{abstract}
We provide the existence of a positive solution for the quasilinear elliptic equation$$
-\operatorname{div}(a(x,|\nabla u|) \nabla u)=f(x, u, \nabla u)
$$

in $\Omega$ under the Dirichlet boundary condition. As a special case $\left(a(x, t)=t^{p-2}\right)$, our equation coincides with the usual $p$-Laplace equation. The solution is established as the limit of a sequence of positive solutions of approximate equations. The positivity of our solution follows from the behavior of $f(x, t \xi)$ as $t$ is small. In this paper, we do not impose the sign condition to the nonlinear term $f$.

MSC: 35 J92; 35P30
\end{abstract}

Keywords: nonhomogeneous elliptic operator; positive solution; the first eigenvalue with weight; approximation

\section{Introduction}

In this paper, we consider the existence of a positive solution for the following quasilinear elliptic equation:

$$
\left\{\begin{array}{l}
-\operatorname{div} A(x, \nabla u)=f(x, u, \nabla u) \text { in } \Omega, \\
u=0 \text { on } \partial \Omega,
\end{array}\right.
$$

where $\Omega \subset \mathbb{R}^{N}$ is a bounded domain with $C^{2}$ boundary $\partial \Omega$. Here, $A: \bar{\Omega} \times \mathbb{R}^{N} \rightarrow \mathbb{R}^{N}$ is a map which is strictly monotone in the second variable and satisfies certain regularity conditions (see the following assumption $(\mathrm{A})$ ). Equation $(P)$ contains the corresponding $p$-Laplacian problem as a special case. However, in general, we do not suppose that this operator is $(p-1)$-homogeneous in the second variable.

Throughout this paper, we assume that the map $A$ and the nonlinear term $f$ satisfy the following assumptions (A) and (f), respectively.

(A) $A(x, y)=a(x,|y|) y$, where $a(x, t)>0$ for all $(x, t) \in \bar{\Omega} \times(0,+\infty)$, and there exist positive constants $C_{0}, C_{1}, C_{2}, C_{3}, 0<t_{0} \leq 1$ and $1<p<\infty$ such that

(i) $A \in C^{0}\left(\bar{\Omega} \times \mathbb{R}^{N}, \mathbb{R}^{N}\right) \cap C^{1}\left(\bar{\Omega} \times\left(\mathbb{R}^{N} \backslash\{0\}\right), \mathbb{R}^{N}\right)$;

(ii) $\left|D_{y} A(x, y)\right| \leq C_{1}|y|^{p-2}$ for every $x \in \bar{\Omega}$, and $y \in \mathbb{R}^{N} \backslash\{0\}$;

(iii) $D_{y} A(x, y) \xi \cdot \xi \geq C_{0}|y|^{p-2}|\xi|^{2}$ for every $x \in \bar{\Omega}, y \in \mathbb{R}^{N} \backslash\{0\}$ and $\xi \in \mathbb{R}^{N}$; 
(iv) $\left|D_{x} A(x, y)\right| \leq C_{2}\left(1+|y|^{p-1}\right)$ for every $x \in \bar{\Omega}, y \in \mathbb{R}^{N} \backslash\{0\}$;

(v) $\left|D_{x} A(x, y)\right| \leq C_{3}|y|^{p-1}(-\log |y|)$ for every $x \in \bar{\Omega}, y \in \mathbb{R}^{N}$ with $0<|y|<t_{0}$.

(f) $f$ is a continuous function on $\Omega \times[0, \infty) \times \mathbb{R}^{N}$ satisfying $f(x, 0, \xi)=0$ for every

$(x, \xi) \in \Omega \times \mathbb{R}^{N}$ and the following growth condition: there exist $1<q<p, b_{1}>0$ and a continuous function $f_{0}$ on $\Omega \times[0, \infty)$ such that

$$
-b_{1}\left(1+t^{q-1}\right) \leq f_{0}(x, t) \leq f(x, t, \xi) \leq b_{1}\left(1+t^{q-1}+|\xi|^{q-1}\right)
$$

for every $(x, t, \xi) \in \Omega \times[0, \infty) \times \mathbb{R}^{N}$.

In this paper, we say that $u \in W_{0}^{1, p}(\Omega)$ is a (weak) solution of $(P)$ if

$$
\int_{\Omega} A(x, \nabla u) \nabla \varphi d x=\int_{\Omega} f(x, u, \nabla u) \varphi d x
$$

for all $\varphi \in W_{0}^{1, p}(\Omega)$.

A similar hypothesis to (A) is considered in the study of quasilinear elliptic problems (see [1, Example 2.2.], [2-5] and also refer to [6, 7] for the generalized $p$-Laplace operators). From now on, we assume that $C_{0} \leq p-1 \leq C_{1}$, which is without any loss of generality as can be seen from assumptions (A)(ii), (iii).

In particular, for $A(x, y)=|y|^{p-2} y$, that is, $\operatorname{div} A(x, \nabla u)$ stands for the usual $p$-Laplacian $\Delta_{p} u$, we can take $C_{0}=C_{1}=p-1$ in (A). Conversely, in the case where $C_{0}=C_{1}=p-1$ holds in (A), by the inequalities in Remark 3(ii) and (iii), we see that $a(x, t)=|t|^{p-2}$ whence $A(x, y)=|y|^{p-2} y$. Hence, our equation contains the $p$-Laplace equation as a special case.

In the case where $f$ does not depend on the gradient of $u$, there are many existence results because our equation has the variational structure $(c f .[1,4,8])$. Although there are a few results for our equation $(P)$ with $f$ including $\nabla u$, we can refer to [7,9] and [10] for the existence of a positive solution in the case of the $(p, q)$-Laplacian or $m$-Laplacian $(1<m<$ $N)$. In particular, in [9] and [7], the nonlinear term $f$ is imposed to be nonnegative. The results in [7] and [10] are applied to the $m$-Laplace equation with an $(m-1)$-superlinear term $f$ w.r.t. $u$. Here, we mention the result in [9] for the $p$-Laplacian. Faria, Miyagaki and Motreanu considered the case where $f$ is $(p-1)$-sublinear w.r.t. $u$ and $\nabla u$, and they supposed that $f(x, u, \nabla u) \geq c u^{r}$ for some $c>0$ and $0<r<p-1$. The purpose of this paper is to remove the sign condition and to admit the condition like $f(x, u, \nabla u) \geq \lambda u^{p-1}+o\left(u^{p-1}\right)$ for large $\lambda>0$ as $u \rightarrow 0+$. Concerning the condition for $f$ as $|u| \rightarrow 0$, Zou in [10] imposed that there exists an $L>0$ satisfying $f(x, u, \nabla u)=L u^{m-1}+o\left(|u|^{m-1}+|\nabla u|^{m-1}\right)$ as $|u|,|\nabla u| \rightarrow 0$ for the $m$-Laplace problem. Hence, we cannot apply the result of [10] and [9] to the case of $f(x, u, \nabla u)=\lambda m(x) u^{p-1}+\left(1-u^{p-1}\right)|\nabla u|^{r-1}+o\left(u^{p-1}\right)$ as $u \rightarrow 0+$ for $1<r<p$ and $m \in L^{\infty}(\Omega)$ (admitting sign changes), but we can do our result if $\lambda>0$ is large.

In [9], the positivity of a solution is proved by the comparison principle. However, since we are not able to do it for our operator in general, after we provide a non-negative and non-trivial solution as a limit of positive approximate solutions (in Section 2), we obtain the positivity of it due to the strong maximum principle for our operator.

\subsection{Statements}

To state our first result, we define a positive constant $A_{p}$ by

$$
A_{p}:=\frac{C_{1}}{p-1}\left(\frac{C_{1}}{C_{0}}\right)^{p-1} \geq 1
$$


which is equal to 1 in the case of $A(x, y)=|y|^{p-2} y$ (i.e., the case of the $p$-Laplacian) because we can choose $C_{0}=C_{1}=p-1$. Then, we introduce the hypothesis (f1) to the function $f_{0}(x, t)$ in (f) as $t$ is small.

(f1) There exist $m \in L^{\infty}(\Omega)$ and $b_{0}>\mu_{1}(m) A_{p}$ such that the Lebesgue measure of $\{x \in \Omega ; m(x)>0\}$ is positive and

$$
\liminf _{t \rightarrow 0+} \frac{f_{0}(x, t)}{t^{p-1}} \geq b_{0} m(x) \quad \text { uniformly in } x \in \Omega,
$$

where $f_{0}$ is the continuous function in (f) and $\mu_{1}(m)$ is the first positive eigenvalue of the $p$-Laplacian with the weight function $m$ obtained by

$$
\mu_{1}(m):=\inf \left\{\int_{\Omega}|\nabla u|^{p} d x ; u \in W_{0}^{1, p}(\Omega) \text { and } \int_{\Omega} m|u|^{p} d x=1\right\} .
$$

Theorem 1 Assume (f1). Then equation $(P)$ has a positive solution $u \in \operatorname{int} P$, where

$$
\begin{aligned}
& P:=\left\{u \in C_{0}^{1}(\bar{\Omega}) ; u(x) \geq 0 \text { in } \Omega\right\}, \\
& \operatorname{int} P:=\left\{u \in C_{0}^{1}(\bar{\Omega}) ; u(x)>0 \text { in } \Omega \text { and } \partial u / \partial v<0 \text { on } \partial \Omega\right\},
\end{aligned}
$$

and $v$ denotes the outward unit normal vector on $\partial \Omega$.

Next, we consider the case where $A$ is asymptotically $(p-1)$-homogeneous near zero in the following sense:

(AH0) There exist a positive function $a_{0} \in C(\bar{\Omega},(0,+\infty))$ and $\tilde{a}_{0}(x, t) \in C(\bar{\Omega} \times[0,+\infty), \mathbb{R})$ such that

$$
\begin{aligned}
& A(x, y)=a_{0}(x)|y|^{p-2} y+\widetilde{a}_{0}(x,|y|) y \quad \text { for every } x \in \Omega, y \in \mathbb{R}^{N} \text { and } \\
& \lim _{t \rightarrow 0+} \frac{\widetilde{a}_{0}(x, t)}{t^{p-2}}=0 \quad \text { uniformly in } x \in \bar{\Omega} .
\end{aligned}
$$

Under (AH0), we can replace the hypothesis (f1) with the following (f2):

(f2) There exist $m \in L^{\infty}(\Omega)$ and $b_{0}>\lambda_{1}(m)$ such that (3) and the Lebesgue measure of $\{x \in \Omega ; m(x)>0\}$ is positive, where $\lambda_{1}(m)$ is the first positive eigenvalue of $-\operatorname{div}\left(a_{0}(x)|\nabla u|^{p-2} \nabla u\right)$ with a weight function $m$ obtained by

$$
\lambda_{1}(m):=\inf \left\{\int_{\Omega} a_{0}(x)|\nabla u|^{p} d x ; u \in W_{0}^{1, p}(\Omega) \text { and } \int_{\Omega} m|u|^{p} d x=1\right\} .
$$

Theorem 2 Assume (AH0) and (f2). Then equation ( $P$ ) has a positive solution $u \in \operatorname{int} P$.

Throughout this paper, we may assume that $f(x, t, \xi)=0$ for every $t \leq 0, x \in \Omega$ and $\xi \in$ $\mathbb{R}^{N}$ because we consider the existence of a positive solution only. In what follows, the norm on $W_{0}^{1, p}(\Omega)$ is given by $\|u\|:=\|\nabla u\|_{p}$, where $\|u\|_{q}$ denotes the usual norm of $L^{q}(\Omega)$ for $u \in L^{q}(\Omega)(1 \leq q \leq \infty)$. Moreover, we denote $u_{ \pm}:=\max \{ \pm u, 0\}$.

\subsection{Properties of the map $A$}

Remark 3 The following assertions hold under condition (A):

(i) for all $x \in \bar{\Omega}, A(x, y)$ is maximal monotone and strictly monotone in $y$;

(ii) $|A(x, y)| \leq \frac{C_{1}}{p-1}|y|^{p-1}$ for every $(x, y) \in \bar{\Omega} \times \mathbb{R}^{N}$; 
(iii) $A(x, y) y \geq \frac{C_{0}}{p-1}|y|^{p}$ for every $(x, y) \in \bar{\Omega} \times \mathbb{R}^{N}$, where $C_{0}$ and $C_{1}$ are the positive constants in (A).

Proposition 4 ([3, Proposition 1]) Let $A: W_{0}^{1, p}(\Omega) \rightarrow W_{0}^{1, p}(\Omega)^{*}$ be a map defined by

$$
\langle A(u), v\rangle=\int_{\Omega} A(x, \nabla u) \nabla v d x
$$

for $u, v \in W_{0}^{1, p}(\Omega)$. Then $A$ is maximal monotone, strictly monotone and has $(S)_{+}$property, that is, any sequence $\left\{u_{n}\right\}$ weakly convergent to $u$ with $\lim _{\sup _{n \rightarrow \infty}}\left\langle A\left(u_{n}\right), u_{n}-u\right\rangle \leq 0$ strongly converges to $u$.

\section{Constructing approximate solutions}

Choose a function $\psi \in P \backslash\{0\}$. In this section, for such $\psi$ and $\varepsilon>0$, we consider the following elliptic equation:

$$
\left\{\begin{array}{l}
-\operatorname{div} A(x, \nabla u)=f(x, u, \nabla u)+\varepsilon \psi(x) \quad \text { in } \Omega \\
u=0 \text { on } \partial \Omega
\end{array}\right.
$$

In [7], the case $\psi \equiv 1$ in the above equation is considered.

Lemma 5 Suppose (f1) or (f2). Then there exists $\lambda_{0}>0$ such that $f(x, t, \xi) t+\lambda_{0} t^{p} \geq 0$ for every $x \in \Omega, t \geq 0$ and $\xi \in \mathbb{R}^{N}$.

Proof From the growth condition of $f_{0}$ and (3), it follows that

$$
f_{0}(x, t) t \geq-b_{0}\|m\|_{\infty} t^{p}-b_{1}^{\prime} t^{p} \quad \text { for every }(x, t) \in \Omega \times[0, \infty)
$$

holds, where $b_{1}^{\prime}$ is a positive constant independent of $(x, t)$. Therefore, for $\lambda_{0} \geq b_{0}\|m\|_{\infty}+$ $b_{1}^{\prime}$, we easily see that $f(x, t, \xi) t+\lambda_{0} t^{p} \geq f_{0}(x, t) t+\lambda_{0} t^{p} \geq 0$ for every $x \in \Omega, t \geq 0$ and $\xi \in \mathbb{R}^{N}$ holds.

Proposition 6 If $u_{\varepsilon} \in W_{0}^{1, p}(\Omega)$ is a non-negative solution of $(P ; \varepsilon)$ for $\varepsilon \geq 0$, then $u_{\varepsilon} \in$ $L^{\infty}(\Omega)$. Moreover, for any $\varepsilon_{0}>0$, there exists a positive constant $D>0$ such that $\left\|u_{\varepsilon}\right\|_{\infty} \leq$ $D \max \left\{1,\left\|u_{\varepsilon}\right\|\right\}$ holds for every $\varepsilon \in\left[0, \varepsilon_{0}\right]$.

Proof Set $\bar{p}^{*}=N p /(N-p)$ if $N>p$, and in the case of $N \leq p, \bar{p}^{*}>p$ is an arbitrarily fixed constant. Let $u_{\varepsilon}$ be a non-negative solution of $(P ; \varepsilon)$ with $0 \leq \varepsilon \leq \varepsilon_{0}$ (some $\varepsilon_{0}>0$ ). For $r>0$, choose a smooth increasing function $\eta(t)$ such that $\eta(t)=t^{r+1}$ if $0 \leq t \leq 1, \eta(t)=$ $d_{0} t$ if $t \geq d_{1}$ and $\eta^{\prime}(t) \geq d_{2}>0$ if $1 \leq t \leq d_{1}$ for some $0<d_{2}<1<d_{0}, d_{1}$. Define $\xi_{M}(u):=$ $M^{r+1} \eta(u / M)$ for $M>1$.

If $u_{\varepsilon} \in L^{r+p}(\Omega)$, then by taking $\xi_{M}\left(u_{\varepsilon}\right)$ as a test function (note that $\eta^{\prime}$ is bounded), we have

$$
\begin{aligned}
& \frac{C_{0}}{p-1} \int_{\Omega}\left|\nabla u_{\varepsilon}\right|^{p} \xi_{M}^{\prime}\left(u_{\varepsilon}\right) d x \\
& \quad \leq \int_{\Omega} A\left(x, \nabla u_{\varepsilon}\right) \nabla u_{\varepsilon} \xi_{M}^{\prime}\left(u_{\varepsilon}\right) d x
\end{aligned}
$$




$$
\begin{aligned}
& =\int_{\Omega}\left(f\left(x, u_{\varepsilon}, \nabla u_{\varepsilon}\right)+\varepsilon \psi\right) \xi_{M}\left(u_{\varepsilon}\right) d x \\
& \leq b_{1} \int_{\Omega}\left(1+u_{\varepsilon}^{q-1}+\varepsilon_{0}\|\psi\|_{\infty}\right) M^{r+1} \eta\left(u_{\varepsilon} / M\right) d x+b_{1} \int_{\Omega}\left|\nabla u_{\varepsilon}\right|^{q-1} \xi_{M}\left(u_{\varepsilon}\right) d x \\
& \leq d_{0} d_{1}\left(2 b_{1}+\varepsilon_{0}\|\psi\|_{\infty}\right)\left(\left\|u_{\varepsilon}\right\|_{r+q}^{r+q}+\left\|u_{\varepsilon}\right\|_{r+1}^{r+1}\right)+b_{1} \int_{\Omega}\left|\nabla u_{\varepsilon}\right|^{q-1} \xi_{M}\left(u_{\varepsilon}\right) d x
\end{aligned}
$$

due to Remark 3(iii) and $M^{r+1} \eta(t / M) \leq d_{0} d_{1} t^{r+1}$. Putting $\beta:=p /(p-q+1)<p$, we see that $\left(\xi_{M}\left(u_{\varepsilon}\right)\right) /\left(\xi_{M}^{\prime}\left(u_{\varepsilon}\right)\right)^{(q-1) / p}=u_{\varepsilon}^{r+1} /\left((r+1) u_{\varepsilon}^{r}\right)^{(q-1) / p} \leq u_{\varepsilon}^{1+r / \beta}$ provided $0<u_{\varepsilon}<M$ (note $r>0)$. Similarly, if $M \leq u_{\varepsilon} \leq d_{1} M$, then $\left(\xi_{M}\left(u_{\varepsilon}\right)\right) /\left(\xi_{M}^{\prime}\left(u_{\varepsilon}\right)\right)^{(q-1) / p} \leq d_{0} d_{1} M^{r+1} /\left(d_{2} M^{r}\right)^{(q-1) / p}=$ $d_{0} d_{1} d_{2}^{(1-q) / p} M^{1+r / \beta} \leq d_{0} d_{1} d_{2}^{(1-q) / p} u_{\varepsilon}^{1+r / \beta}$, and if $u_{\varepsilon}>d_{1} M$, then $\left(\xi_{M}\left(u_{\varepsilon}\right)\right) /\left(\xi_{M}^{\prime}\left(u_{\varepsilon}\right)\right)^{(q-1) / p}=$ $d_{0}^{1 / \beta} M^{r / \beta} u_{\varepsilon} \leq d_{0}^{1 / \beta} u_{\varepsilon}^{1+r / \beta}$ (note $d_{1}>1$ ). Thus, according to Young's inequality, for every $\delta>0$, there exists $C_{\delta}>0$ such that

$$
\begin{aligned}
\int_{\Omega}\left|\nabla u_{\varepsilon}\right|^{q-1} \xi_{M}\left(u_{\varepsilon}\right) d x & \leq \delta \int_{\Omega}\left|\nabla u_{\varepsilon}\right|^{p} \xi_{M}^{\prime}\left(u_{\varepsilon}\right) d x+C_{\delta} \int_{u_{\varepsilon}>0} \frac{\left(\xi_{M}\left(u_{\varepsilon}\right)\right)^{\beta}}{\left(\xi_{M}^{\prime}\left(u_{\varepsilon}\right)\right)^{(q-1) \beta / p}} d x \\
& \leq \delta \int_{\Omega}\left|\nabla u_{\varepsilon}\right|^{p} \xi_{M}^{\prime}\left(u_{\varepsilon}\right) d x+C_{\delta} d_{3} \int_{\Omega} u_{\varepsilon}^{r+\beta} d x,
\end{aligned}
$$

where $\beta:=p /(p-q+1)<p$ and $d_{3}=\max \left\{d_{0} d_{1} d_{2}^{(1-q) / p}, d_{0}^{1 / \beta}\right\}(>1)$. As a result, because of $r+p>r+q, r+\beta$, according to Hölder's inequality and the monotonicity of $t^{r}$ with respect to $r$ on $[1, \infty)$, taking a $0<\delta<C_{0} / b_{1}(p-1)$ and setting $u_{\varepsilon}^{M}(x):=\min \left\{u_{\varepsilon}(x), M\right\}$, we obtain

$$
\begin{aligned}
b_{4}\left(r^{\prime}\right)^{p} \max \left\{1,\left\|u_{\varepsilon}\right\|_{r+p}^{r+p}\right\} & \geq\left(r^{\prime}\right)^{p} \int_{\Omega}\left|\nabla u_{\varepsilon}\right|^{p} \xi_{M}^{\prime}\left(u_{\varepsilon}\right) d x \geq\left(r^{\prime}\right)^{p} \int_{\Omega}\left|\nabla u_{\varepsilon}^{M}\right|^{p}\left(u_{\varepsilon}^{M}\right)^{r} d x \\
& =\left\|\left(u_{\varepsilon}^{M}\right)^{r^{\prime}}\right\|^{p} \geq C_{*}\left\|\left(u_{\varepsilon}^{M}\right)^{r^{\prime}}\right\|_{\bar{p}^{*}}^{p}=C_{*}\left\|u_{\varepsilon}^{M}\right\|_{\bar{p}^{*} r^{\prime}}^{r+p}
\end{aligned}
$$

provided $u_{\varepsilon} \in L^{r+p}(\Omega)$ by (8) and (9), where $r^{\prime}=1+r / p, C_{*}$ comes from the continuous embedding of $W_{0}^{1, p}(\Omega)$ into $L^{\bar{p}^{*}}(\Omega)$ and $d_{4}$ is a positive constant independent of $u_{\varepsilon}$, $\varepsilon$ and $r$. Consequently, Moser's iteration process implies our conclusion. In fact, we define a sequence $\left\{r_{m}\right\}_{m}$ by $r_{0}:=\bar{p}^{*}-p$ and $r_{m+1}:=\bar{p}^{*}\left(p+r_{m}\right) / p-p$. Then, we see that $u_{\varepsilon} \in$ $L^{\bar{p}^{*}\left(p+r_{m}\right) / p}(\Omega)=L^{p+r_{m+1}}(\Omega)$ holds if $u_{\varepsilon} \in L^{p+r_{m}}(\Omega)$ by applying Fatou's lemma to (10) and letting $M \rightarrow \infty$. Here, we also see $r_{m+1}=\bar{p}^{*} r_{m} / p+\bar{p}^{*}-p \geq\left(\bar{p}^{*} / p\right)^{m+1} r_{0} \rightarrow \infty$ as $m \rightarrow \infty$. Therefore, by the same argument as in Theorem $C$ in [4], we can obtain $u_{\varepsilon} \in L^{\infty}(\Omega)$ and $\left\|u_{\varepsilon}\right\|_{\infty} \leq D \max \left\{1,\left\|u_{\varepsilon}\right\|\right\}$ for some positive constant $D$ independent of $u_{\varepsilon}$ and $\varepsilon$.

Lemma 7 Suppose (f1) or (f2). If $u_{\varepsilon} \in W_{0}^{1, p}(\Omega)$ is a solution of $(P ; \varepsilon)$ for $\varepsilon>0$, then $u_{\varepsilon} \in$ int $P$.

Proof Taking $-\left(u_{\varepsilon}\right)_{-}$as a test function in $(P ; \varepsilon)$, we have

$$
\frac{C_{0}}{p-1}\left\|\nabla\left(u_{\varepsilon}\right)_{-}\right\|_{p}^{p} \leq \int_{\Omega} A\left(x, \nabla u_{\varepsilon}\right)\left(-\nabla\left(u_{\varepsilon}\right)_{-}\right) d x=-\varepsilon \int_{\Omega} \psi\left(u_{\varepsilon}\right)_{-} d x \leq 0
$$

because of $f(x, t, \xi)=0$ if $t \leq 0$ and by Remark 3(iii). Hence, $u_{\varepsilon} \geq 0$ follows. Because Proposition 6 guarantees that $u_{\varepsilon} \in L^{\infty}(\Omega)$, we have $u_{\varepsilon} \in C_{0}^{1, \alpha}(\bar{\Omega})$ (for some $0<\alpha<1$ ) by the regularity result in [11]. Note that $u_{\varepsilon} \not \equiv 0$ because of $\varepsilon>0$ and $\psi \neq \equiv 0$. In addition, Lemma 5 implies the existence of $\lambda_{0}>0$ such that $-\operatorname{div} A\left(x, \nabla u_{\varepsilon}\right)+\lambda_{0} u_{\varepsilon}^{p-1} \geq 0$ in the distribution sense. 
Therefore, according to Theorem A and Theorem B in [4], $u_{\varepsilon}>0$ in $\Omega$ and $\partial u_{\varepsilon} / \partial v<0$ on $\partial \Omega$, namely, $u_{\varepsilon} \in \operatorname{int} P$.

The following result can be shown by the same argument as in [9, Theorem 3.1].

Proposition 8 Suppose (f1) or (f2). Then, for every $\varepsilon>0,(P ; \varepsilon)$ has a positive solution $u_{\varepsilon} \in$ int $P$.

Proof Fix any $\varepsilon>0$ and let $\left\{e_{1}, \ldots, e_{m}, \ldots\right\}$ be a Schauder basis of $W_{0}^{1, p}(\Omega)$ (refer to [12] for the existence). For each $m \in \mathbb{N}$, we define the $m$-dimensional subspace $V_{m}$ of $W_{0}^{1, p}(\Omega)$ by $V_{m}:=\operatorname{lin}$. sp. $\left\{e_{1}, \ldots, e_{m}\right\}$. Moreover, set a linear isomorphism $T_{m}: \mathbb{R}^{m} \rightarrow V_{m}$ by $T_{m}\left(\xi_{1}, \ldots, \xi_{m}\right):=\sum_{i=1}^{m} \xi_{i} e_{i} \in V_{m}$, and let $T_{m}^{*}: V_{m}^{*} \rightarrow\left(\mathbb{R}^{m}\right)^{*}$ be a dual map of $T_{m}$. By identifying $\mathbb{R}^{m}$ and $\left(\mathbb{R}^{m}\right)^{*}$, we may consider that $T_{m}^{*}$ maps from $V_{m}^{*}$ to $\mathbb{R}^{m}$. Define maps $A_{m}$ and $B_{m}$ from $V_{m}$ to $V_{m}^{*}$ as follows:

$$
\left\langle A_{m}(u), v\right\rangle:=\int_{\Omega} A(x, \nabla u) \nabla v d x \text { and }\left\langle B_{m}(u), v\right\rangle:=\int_{\Omega} f(x, u, \nabla u) v d x+\varepsilon \int_{\Omega} \psi v d x
$$

for $u, v \in V_{m}$. We claim that for every $m \in \mathbb{N}$, there exists $u_{m} \in V_{m}$ such that $A_{m}\left(u_{m}\right)-$ $B_{m}\left(u_{m}\right)=0$ in $V_{m}^{*}$. Indeed, by the growth condition of $f$, Remark 3(iii) and Hölder's inequality, we easily have

$$
\begin{aligned}
& \left\langle A_{m}(u)-B_{m}(u), u\right\rangle \\
& \quad \geq \frac{C_{0}}{p-1}\|u\|^{p}-b_{1}\left(\|u\|_{1}+\|u\|_{q}^{q}+\|\nabla u\|_{p}^{q-1}\|u\|_{\beta}\right)-\varepsilon\|\psi\|_{\infty}\|u\|_{1}
\end{aligned}
$$

for every $u \in V_{m}$, where $\beta=p /(p-q+1)<p$. This implies that $A_{m}-B_{m}$ is coercive on $V_{m}$ by $q<p$. Set a homotopy $H_{m}(t, y):=t y+(1-t) T_{m}^{*}\left(A_{m}\left(T_{m}(y)\right)-B_{m}\left(T_{m}(y)\right)\right)$ for $t \in[0,1]$ and $y \in \mathbb{R}^{m}$. By recalling that $A_{m}-B_{m}$ is coercive on $V_{m}$, we see that there exists an $R>0$ such that $\left(H_{m}(t, y), y\right)>0$ for every $t \in[0,1]$ and $|y| \geq R$ because $\|\cdot\|$ and the norm of $\mathbb{R}^{m}$ are equivalent on $V_{m}$. Therefore, we have

$$
\begin{aligned}
1 & =\operatorname{deg}\left(I_{m}, B_{R}(0), 0\right)=\operatorname{deg}\left(H_{m}(1, \cdot), B_{R}(0), 0\right) \\
& =\operatorname{deg}\left(H_{m}(0, \cdot), B_{R}(0), 0\right)=\operatorname{deg}\left(T_{m}^{*} \circ\left(A_{m}-B_{m}\right) \circ T_{m}, B_{R}(0), 0\right),
\end{aligned}
$$

where $I_{m}$ is the identity map on $\mathbb{R}^{m}, B_{R}(0):=\left\{y \in \mathbb{R}^{m} ;|y|<R\right\}$ and $\operatorname{deg}(g, B, 0)$ denotes the degree on $\mathbb{R}^{m}$ for a continuous map $g: B \rightarrow \mathbb{R}^{m}$ (cf. [13]). Hence, this yields the existence of $y_{m} \in \mathbb{R}^{m}$ such that $\left(T_{m}^{*} \circ\left(A_{m}-B_{m}\right) \circ T_{m}\right)\left(y_{m}\right)=0$, and so the desired $u_{m}$ is obtained by setting $u_{m}=T_{m}\left(y_{m}\right) \in V_{m}$ since $T_{m}^{*}$ is injective.

Because (11) with $u=u_{m} \in W_{0}^{1, p}(\Omega)$ leads to the boundedness of $\left\|u_{m}\right\|$ by $q<p$, we may assume, by choosing a subsequence, that $u_{m}$ converges to some $u_{0}$ weakly in $W_{0}^{1, p}(\Omega)$ and strongly in $L^{p}(\Omega)$. Let $P_{m}$ be a natural projection onto $V_{m}$, that is, $P_{m} u=\sum_{i=1}^{m} \xi_{i} e_{i}$ for $u=$ $\sum_{i=1}^{\infty} \xi_{i} e_{i}$. Since $u_{m}, P_{m} u_{0} \in V_{m}$ and $A_{m}\left(u_{m}\right)-B_{m}\left(u_{m}\right)=0$ in $V_{m}^{*}$, by noting that $A_{m}=A$ on $V_{m}$ for a map $A$ defined in Proposition 4, we obtain

$$
\begin{aligned}
& \left\langle A\left(u_{m}\right), u_{m}-u_{0}\right\rangle+\left\langle A\left(u_{m}\right), u_{0}-P_{m} u_{0}\right\rangle \\
& \quad=\left\langle A_{m}\left(u_{m}\right), u_{m}-P_{m} u_{0}\right\rangle=\left\langle B_{m}\left(u_{m}\right), u_{m}-P_{m} u_{0}\right\rangle
\end{aligned}
$$




$$
\begin{aligned}
= & \int_{\Omega}\left(f\left(x, u_{m}, \nabla u_{m}\right)+\varepsilon \psi\right)\left(u_{m}-u_{0}\right) d x \\
& +\int_{\Omega}\left(f\left(x, u_{m}, \nabla u_{m}\right)+\varepsilon \psi\right)\left(u_{0}-P_{m} u_{0}\right) d x \rightarrow 0
\end{aligned}
$$

as $m \rightarrow \infty$, where we use the boundedness of $\left\|u_{m}\right\|$, the growth condition of $f$ and $u_{m} \rightarrow$ $u_{0}$ in $L^{p}(\Omega)$. In addition, since $\left\|A\left(u_{m}\right)\right\|_{W_{0}^{1, p}(\Omega)^{*}}$ is bounded, by the boundedness of $\left\|u_{m}\right\|$, we see that $\left\langle A\left(u_{m}\right), u_{0}-P_{m} u_{0}\right\rangle \rightarrow 0$ as $m \rightarrow \infty$, whence $\left\langle A\left(u_{m}\right), u_{m}-u_{0}\right\rangle \rightarrow 0$ as $m \rightarrow$ $\infty$ holds. As a result, it follows from the $(S)_{+}$property of $A$ that $u_{m} \rightarrow u_{0}$ in $W_{0}^{1, p}(\Omega)$ as $m \rightarrow \infty$.

Finally, we shall prove that $u_{0}$ is a solution of $(P ; \varepsilon)$. Fix any $l \in \mathbb{N}$ and $\varphi \in V_{l}$. For each $m \geq l$, by letting $m \rightarrow \infty$ in $\left\langle A_{m}\left(u_{m}\right), \varphi\right\rangle=\left\langle B_{m}\left(u_{m}\right), \varphi\right\rangle$, we have

$$
\int_{\Omega} A\left(x, \nabla u_{0}\right) \nabla \varphi d x=\int_{\Omega} f\left(x, u_{0}, \nabla u_{0}\right) \varphi d x+\varepsilon \int_{\Omega} \psi \varphi d x
$$

Since $l$ is arbitrary, (12) holds for every $\varphi \in \bigcup_{l \geq 1} V_{l}$. Moreover, the density of $\bigcup_{l \geq 1} V_{l}$ in $W_{0}^{1, p}(\Omega)$ guarantees that (12) holds for every $\varphi \in W_{0}^{1, p}(\Omega)$. This means that $u_{0}$ is a solution of $(P ; \varepsilon)$. Consequently, our conclusion $u_{0} \in \operatorname{int} P$ follows from Lemma 7 .

\section{Proof of theorems}

Lemma 9 Let $\varphi, u \in \operatorname{int} P$. Then

$$
\int_{\Omega} A(x, \nabla u) \nabla\left(\frac{\varphi^{p}}{u^{p-1}}\right) d x \leq A_{p}\|\nabla \varphi\|_{p}^{p}
$$

holds, where $A_{p}$ is the positive constant defined by (2).

Proof Because of $\varphi, u \in \operatorname{int} P$, there exist $\delta_{1}>\delta_{2}>0$ such that $\delta_{1} u \geq \varphi \geq \delta_{2} u$ in $\bar{\Omega}$. Thus, $\delta_{1} \geq \varphi / u \geq \delta_{2}$ and $1 / \delta_{2} \geq u / \varphi \geq 1 / \delta_{1}$ in $\Omega$. Hence, $u / \varphi, \varphi / u \in L^{\infty}(\Omega)$ hold. Therefore, we have

$$
\begin{aligned}
A(x, \nabla u) \nabla\left(\frac{\varphi^{p}}{u^{p-1}}\right)= & p\left(\frac{\varphi}{u}\right)^{p-1} A(x, \nabla u) \nabla \varphi-(p-1)\left(\frac{\varphi}{u}\right)^{p} A(x, \nabla u) \nabla u \\
\leq & \frac{p C_{1}}{p-1}\left(\frac{\varphi}{u}\right)^{p-1}|\nabla u|^{p-1}|\nabla \varphi|-C_{0}\left(\frac{\varphi}{u}\right)^{p}|\nabla u|^{p} \\
= & \left\{\left(\frac{p C_{0}}{p-1}\right)^{1 / p} \frac{\varphi}{u}|\nabla u|\right\}^{p-1}\left(\frac{p}{p-1}\right)^{1 / p} C_{1} C_{0}^{(1-p) / p}|\nabla \varphi| \\
& -C_{0}\left(\frac{\varphi}{u}\right)^{p}|\nabla u|^{p} \leq A_{p}|\nabla \varphi|^{p}
\end{aligned}
$$

in $\Omega$ by (ii) and (iii) in Remark 3 and Young's inequality.

Lemma 10 Assume that $a_{0} \in C(\bar{\Omega},[0, \infty))$ and let $\varphi, u \in \operatorname{int} P$. Then

$$
\int_{\Omega} a_{0}(x)|\nabla \varphi|^{p-2} \nabla \varphi \nabla\left(\frac{\varphi^{p}-u^{p}}{\varphi^{p-1}}\right) d x-\int_{\Omega} a_{0}(x)|\nabla u|^{p-2} \nabla u \nabla\left(\frac{\varphi^{p}-u^{p}}{u^{p-1}}\right) d x \geq 0
$$

holds. 
Proof First, we note that $u / \varphi, \varphi / u \in L^{\infty}(\Omega)$ hold by the same reason as in Lemma 9. Applying Young's inequality to the second term of the right-hand side in (14) (refer to (13) with $C_{0}=C_{1}=p-1$ ), we obtain

$$
\begin{aligned}
& a_{0}(x)|\nabla \varphi|^{p-2} \nabla \varphi \nabla\left(\frac{\varphi^{p}-u^{p}}{\varphi^{p-1}}\right) \\
& \quad \geq a_{0}(x)\left(|\nabla \varphi|^{p}-p\left(\frac{u}{\varphi}\right)^{p-1}|\nabla \varphi|^{p-1}|\nabla u|+(p-1)\left(\frac{u}{\varphi}\right)^{p}|\nabla \varphi|^{p}\right) \\
& \quad \geq a_{0}(x)\left(|\nabla \varphi|^{p}-|\nabla u|^{p}\right)
\end{aligned}
$$

in $\Omega$. Similarly, we also have

$$
a_{0}(x)|\nabla u|^{p-2} \nabla u \nabla\left(\frac{\varphi^{p}-u^{p}}{u^{p-1}}\right) \leq a_{0}(x)\left(|\nabla \varphi|^{p}-|\nabla u|^{p}\right) \quad \text { in } \Omega .
$$

The conclusion follows from (15) and (16).

Under (f1) or (f2), we denote a solution $u_{\varepsilon} \in \operatorname{int} P$ of $(P ; \varepsilon)$ for each $\varepsilon>0$ obtained by Proposition 8.

Lemma 11 Assume (f1) or (f2). Let $I:=(0,1]$. Then $\left\{u_{\varepsilon}\right\}_{\varepsilon \in I}$ is bounded in $W_{0}^{1, p}(\Omega)$.

Proof Taking $u_{\varepsilon}$ as a test function in $(P ; \varepsilon)$, we have

$$
\begin{aligned}
\frac{C_{0}}{p-1}\left\|\nabla u_{\varepsilon}\right\|_{p}^{p} & \leq \int_{\Omega} A\left(x, \nabla u_{\varepsilon}\right) \nabla u_{\varepsilon} d x=\int_{\Omega} f\left(x, u_{\varepsilon}, \nabla u_{\varepsilon}\right) u_{\varepsilon} d x+\varepsilon \int_{\Omega} \psi u_{\varepsilon} d x \\
& \leq b_{1}\left(\left\|u_{\varepsilon}\right\|_{1}+\left\|u_{\varepsilon}\right\|_{q}^{q}+\left\|\nabla u_{\varepsilon}\right\|_{p}^{q-1}\left\|u_{\varepsilon}\right\|_{\beta}\right)+\|\psi\|_{\infty}\left\|u_{\varepsilon}\right\|_{1} \\
& \leq b_{1}^{\prime}\left(\left\|u_{\varepsilon}\right\|+\left\|u_{\varepsilon}\right\|^{q}\right)
\end{aligned}
$$

by Remark 3(iii), the growth condition of $f$, Hölder's inequality and the continuity of the embedding of $W_{0}^{1, p}(\Omega)$ into $L^{p}(\Omega)$, where $\beta=p /(p-q+1)(<p)$ and $b_{1}^{\prime}$ is a positive constant independent of $u_{\varepsilon}$. Because of $q<p$, this yields the boundedness of $\left\|u_{\varepsilon}\right\|\left(=\left\|\nabla u_{\varepsilon}\right\|_{p}\right)$.

Lemma 12 Assume (f1) or (f2). Then $\left|\nabla u_{\varepsilon}\right| / u_{\varepsilon} \in L^{p}(\Omega)$ and $\left\|\left|\nabla u_{\varepsilon}\right| / u_{\varepsilon}\right\|_{p}^{p} \leq \lambda_{0}|\Omega| / C_{0}$ hold for every $\varepsilon>0$, where $|\Omega|$ denotes the Lebesgue measure of $\Omega$, and where $C_{0}$ and $\lambda_{0}$ are positive constants as in (A) and Lemma 5, respectively.

Proof Fix any $\varepsilon>0$ and choose any $\rho>0$. By taking $\left(u_{\varepsilon}+\rho\right)^{1-p}$ as a test function, we obtain

$$
\begin{aligned}
(1-p) \int_{\Omega} \frac{A\left(x, \nabla u_{\varepsilon}\right) \nabla u_{\varepsilon}}{\left(u_{\varepsilon}+\rho\right)^{p}} d x & =\int_{\Omega} \frac{f\left(x, u_{\varepsilon}, \nabla u_{\varepsilon}\right)+\varepsilon \psi}{\left(u_{\varepsilon}+\rho\right)^{p-1}} d x \geq-\lambda_{0} \int_{\Omega} \frac{u_{\varepsilon}^{p-1}}{\left(u_{\varepsilon}+\rho\right)^{p-1}} d x \\
& \geq-\lambda_{0}|\Omega|
\end{aligned}
$$

by Lemma 5 and $\varepsilon \psi \geq 0$. On the other hand, by Remark 3(iii) and $1-p<0$, we have

$$
(1-p) \int_{\Omega} \frac{A\left(x, \nabla u_{\varepsilon}\right) \nabla u_{\varepsilon}}{\left(u_{\varepsilon}+\rho\right)^{p}} d x \leq-C_{0} \int_{\Omega} \frac{\left|\nabla u_{\varepsilon}\right|^{p}}{\left(u_{\varepsilon}+\rho\right)^{p}} d x
$$


Therefore, (17) and (18) imply the inequality $\int_{\Omega}\left|\nabla u_{\varepsilon}\right|^{p} /\left(u_{\varepsilon}+\rho\right)^{p} d x \leq \lambda_{0}|\Omega| / C_{0}$ for every $\rho>0$. As a result, by letting $\rho \rightarrow 0+$, our conclusion is shown.

Lemma 13 Assume (f2) and (AH0). Let $\varphi \in \operatorname{int} P$. If $u_{\varepsilon} \rightarrow 0$ in $C_{0}^{1}(\bar{\Omega})$ as $\varepsilon \rightarrow 0+$, then

$$
\lim _{\varepsilon \rightarrow 0+}\left|\int_{\Omega} \tilde{a}_{0}\left(x,\left|\nabla u_{\varepsilon}\right|\right) \nabla u_{\varepsilon} \nabla\left(\frac{\varphi^{p}-u_{\varepsilon}^{p}}{u_{\varepsilon}^{p-1}}\right) d x\right|=0
$$

holds, where $\widetilde{a}_{0}$ is a continuous function as in (AH0).

Proof Note that $u_{\varepsilon} / \varphi, \varphi / u_{\varepsilon} \in L^{\infty}(\Omega)$ hold (as in the proof of Lemma 9). Because we easily see that $\left.\left|\int_{\Omega} \widetilde{a}_{0}(x,|\nabla u|)\right| \nabla u\right|^{2} d x \mid \leq C\|\nabla u\|_{p}^{p}$ for every $u \in W_{0}^{1, p}(\Omega)$ with some $C>0$ independent of $u$ (see (6)), it is sufficient to show $\left|\int_{\Omega} \widetilde{a}_{0}\left(x,\left|\nabla u_{\varepsilon}\right|\right) \nabla u_{\varepsilon} \nabla\left(\varphi^{p} / u_{\varepsilon}^{p-1}\right) d x\right| \rightarrow 0$ as $\varepsilon \rightarrow 0+$. Here, we fix any $\delta>0$. By the property of $\widetilde{a}_{0}$ (see (6)) and because we are assuming that $u_{\varepsilon} \rightarrow 0$ in $C_{0}^{1}(\bar{\Omega})$ as $\varepsilon \rightarrow 0+$, we have $\left|\widetilde{a}_{0}\left(x,\left|\nabla u_{\varepsilon}\right|\right)\right| \leq \delta\left|\nabla u_{\varepsilon}\right|^{p-2}$ for every $x \in \Omega$ provided sufficiently small $\varepsilon>0$. Therefore, for such sufficiently small $\varepsilon>0$, we obtain

$$
\begin{aligned}
& \left|\int_{\Omega} \tilde{a}_{0}\left(x,\left|\nabla u_{\varepsilon}\right|\right) \nabla u_{\varepsilon} \nabla\left(\frac{\varphi^{p}}{u_{\varepsilon}^{p-1}}\right) d x\right| \\
& \quad \leq p \int_{\Omega} \frac{\left|\tilde{a}_{0}\left(x,\left|\nabla u_{\varepsilon}\right|\right)\right|\left|\nabla u_{\varepsilon}\right||\nabla \varphi| \varphi^{p-1}}{u_{\varepsilon}^{p-1}} d x+(p-1) \int_{\Omega} \frac{\left|\tilde{a}_{0}\left(x,\left|\nabla u_{\varepsilon}\right|\right)\right|\left|\nabla u_{\varepsilon}\right|^{2} \varphi^{p}}{u_{\varepsilon}^{p}} d x \\
& \quad \leq \delta\|\varphi\|_{C_{0}^{1}(\bar{\Omega})}^{p}\left\{p \int_{\Omega}\left(\frac{\left|\nabla u_{\varepsilon}\right|}{u_{\varepsilon}}\right)^{p-1} d x+(p-1) \int_{\Omega}\left(\frac{\left|\nabla u_{\varepsilon}\right|}{u_{\varepsilon}}\right)^{p} d x\right\} \\
& \quad \leq \delta\|\varphi\|_{C_{0}^{1}(\bar{\Omega})}^{p}|\Omega|\left(p\left(\lambda_{0} / C_{0}\right)^{1-1 / p}+(p-1)\left(\lambda_{0} / C_{0}\right)\right)
\end{aligned}
$$

because of $\left|\nabla u_{\varepsilon}\right| / u_{\varepsilon} \in L^{p}(\Omega)$ by Lemma 12 . Since $\delta>0$ is arbitrary, our conclusion is shown.

\subsection{Proof of main results}

\section{Proof of Theorems}

Let $\varepsilon \in(0,1]$. Due to Proposition 6 and Lemma 11, we have $\left\|u_{\varepsilon}\right\|_{\infty} \leq M$ for some $M>0$ independent of $\varepsilon \in(0,1]$. Hence, there exist $M^{\prime}>0$ and $0<\alpha<1$ such that $u_{\varepsilon} \in C_{0}^{1, \alpha}(\bar{\Omega})$ and $\left\|u_{\varepsilon}\right\|_{C_{0}^{1, \alpha}(\bar{\Omega})} \leq M^{\prime}$ for every $\varepsilon \in(0,1]$ by the regularity result in [11]. Because the embedding of $C_{0}^{1, \alpha}(\bar{\Omega})$ into $C_{0}^{1}(\bar{\Omega})$ is compact and by $u_{\varepsilon} \in \operatorname{int} P$, there exists a sequence $\left\{\varepsilon_{n}\right\}$ and $u_{0} \in P$ such that $\varepsilon_{n} \rightarrow 0+$ and $u_{n}:=u_{\varepsilon_{n}} \rightarrow u_{0}$ in $C_{0}^{1}(\bar{\Omega})$ as $n \rightarrow \infty$. If $u_{0} \neq 0$ occurs, then $u_{0} \in$ $\operatorname{int} P$ by the same reason as in Lemma 7 , and hence our conclusion is proved. Now, we shall prove $u_{0} \neq 0$ by contradiction for each theorem. So, we suppose that $u_{0}=0$, whence $u_{n} \rightarrow 0$ in $C_{0}^{1}(\bar{\Omega})$ as $n \rightarrow \infty$.

Proof of Theorem 1 Let $\varphi \in \operatorname{int} P$ be an eigenfunction corresponding to the first positive eigenvalue $\mu_{1}(m)(c f .[14,15]$, it is well known that we can obtain $\varphi$ as the minimizer of (4)), namely, $\varphi$ is a positive solution of $-\Delta_{p} u=\mu_{1}(m) m(x)|u|^{p-2} u$ in $\Omega$ and $u=0$ on $\partial \Omega$. Since $p$-Laplacian is $(p-1)$-homogeneous, we may assume that $\varphi$ satisfies $\int_{\Omega} m(x) \varphi^{p} d x=1$, and hence $\|\nabla \varphi\|_{p}^{p}=\mu_{1}(m) \int_{\Omega} m(x) \varphi^{p} d x=\mu_{1}(m)$ holds by taking $\varphi$ as a test function. Choose $\rho>0$ satisfying $b_{0}-A_{p} \mu_{1}(m)>\rho\|\varphi\|_{p}^{p}$ (note that $b_{0}-A_{p} \mu_{1}(m)>0$ as in (f1)). Due to (f1), there exists a $\delta>0$ such that $f_{0}(x, t) \geq\left(b_{0} m(x)-\rho\right) t^{p-1}$ for every $0 \leq t \leq \delta$ and $x \in \Omega$. Since 
we are assuming $u_{n} \rightarrow 0$ in $C_{0}^{1}(\bar{\Omega})$ as $n \rightarrow \infty,\left\|u_{n}\right\|_{\infty} \leq \delta$ occurs for sufficiently large $n$. Then, for such sufficiently large $n$, according to Lemma 9 , (1) and $\psi \geq 0$, we obtain

$$
\begin{aligned}
A_{p} \mu_{1}(m) & =A_{p}\|\nabla \varphi\|_{p}^{p} \geq \int_{\Omega} A\left(x, \nabla u_{n}\right) \nabla\left(\frac{\varphi^{p}}{u_{n}^{p-1}}\right) d x=\int_{\Omega} \frac{f\left(x, u_{n}, \nabla u_{n}\right)+\varepsilon \psi}{u_{n}^{p-1}} \varphi^{p} d x \\
& \geq \int_{\Omega} \frac{f_{0}\left(x, u_{n}\right)}{u_{n}^{p-1}} \varphi^{p} d x \geq b_{0} \int_{\Omega} m(x) \varphi^{p} d x-\rho\|\varphi\|_{p}^{p}=b_{0}-\rho\|\varphi\|_{p}^{p}>A_{p} \mu_{1}(m) .
\end{aligned}
$$

This is a contradiction.

Proof of Theorem 2 Since $\infty>\sup _{x \in \Omega} a_{0}(x) \geq \inf _{x \in \Omega} a_{0}(x)>0$ holds, by the standard argument as in the $p$-Laplacian, we see that $\lambda_{1}(m)>0$ and it is the first positive eigenvalue of $-\operatorname{div}\left(a_{0}(x)|\nabla u|^{p-2} \nabla u\right)=\lambda m(x)|u|^{p-2} u$ in $\Omega$ and $u=0$ on $\partial \Omega$. Therefore, by the wellknown argument, there exists a positive eigenfunction $\varphi_{1} \in \operatorname{int} P$ corresponding to $\lambda_{1}(m)$ (we can obtain $\varphi_{1}$ as the minimizer of (7)). Hence, by taking $\varphi_{1}$ as a test function, we have $0<\int_{\Omega} a_{0}(x)\left|\nabla \varphi_{1}\right|^{p} d x=\lambda_{1}(m) \int_{\Omega} m(x) \varphi_{1}^{p} d x$. Thus, $\int_{\Omega} m(x) \varphi_{1}^{p} d x>0$ follows. Because $u_{n} \in \operatorname{int} P$ is a solution of $\left(P ; \varepsilon_{n}\right)$ and $\varphi_{1} \in \operatorname{int} P$ is an eigenfunction corresponding to $\lambda_{1}(m)$, according to Lemma 11 and Lemma 13 (note $A(x, y)=a_{0}|y|^{p-2} y+\tilde{a}_{0}(x,|y|) y$ as in (AH0)), we obtain

$$
\begin{aligned}
0 \leq & \int_{\Omega} a_{0}(x)\left|\nabla \varphi_{1}\right|^{p-2} \nabla \varphi_{1} \nabla\left(\frac{\varphi_{1}^{p}-u_{n}^{p}}{\varphi_{1}^{p-1}}\right) d x-\int_{\Omega} a_{0}(x)\left|\nabla u_{n}\right|^{p-2} \nabla u_{n} \nabla\left(\frac{\varphi_{1}^{p}-u_{n}^{p}}{u_{n}^{p-1}}\right) d x \\
\leq & \lambda_{1}(m) \int_{\Omega} m\left(\varphi_{1}^{p}-u_{n}^{p}\right) d x-\int_{\Omega} \frac{f_{0}\left(x, u_{n}\right)}{u_{n}^{p-1}} \varphi_{1}^{p} d x \\
& +\int_{\Omega} \tilde{a}_{0}\left(x,\left|\nabla u_{n}\right|\right) \nabla u_{n} \nabla\left(\frac{\varphi_{1}^{p}-u_{n}^{p}}{u_{n}^{p-1}}\right) d x+\int_{\Omega} f\left(x, u_{n}, \nabla u_{n}\right) u_{n} d x+\varepsilon_{n} \int_{\Omega} \psi u_{n} d x \\
= & -\int_{\Omega}\left(\frac{f_{0}\left(x, u_{n}\right)}{u_{n}^{p-1}}-b_{0} m(x)\right) \varphi_{1}^{p} d x-\left(b_{0}-\lambda_{1}(m)\right) \int m(x) \varphi_{1}^{p} d x+o(1)
\end{aligned}
$$

as $n \rightarrow \infty$ since we are assuming $u_{n} \rightarrow 0$ in $C_{0}^{1}(\bar{\Omega})$, where we use the facts that $\psi \geq 0$ and $\varphi_{1}>0$ in $\Omega$. Furthermore, by Fatou's lemma and (3), we have

$$
\liminf _{n \rightarrow \infty} \int_{\Omega}\left(\frac{f_{0}\left(x, u_{n}\right)}{u_{n}^{p-1}}-b_{0} m(x)\right) \varphi_{1}^{p} d x \geq 0
$$

As a result, by taking a limit superior with respect to $n$ in (19), we have $0 \leq-\left(b_{0}-\right.$ $\left.\lambda_{1}(m)\right) \int m(x) \varphi_{1}^{p} d x<0$. This is a contradiction.

\section{Competing interests}

The author declares that she has no competing interests.

\section{Acknowledgements}

The author would like to express her sincere thanks to Professor Shizuo Miyajima for helpful comments and encouragement. The author thanks Professor Dumitru Motreanu for giving her the opportunity of this work. The author thanks referees for their helpful comments. 


\section{References}

1. Motreanu, D, Papageorgiou, NS: Multiple solutions for nonlinear Neumann problems driven by a nonhomogeneous differential operator. Proc. Am. Math. Soc. 139, 3527-3535 (2011)

2. Damascelli, L: Comparison theorems for some quasilinear degenerate elliptic operators and applications to symmetry and monotonicity results. Ann. Inst. Henri Poincaré 15, 493-516 (1998)

3. Motreanu, D, Motreanu, VV, Papageorgiou, NS: Multiple constant sign and nodal solutions for nonlinear Neumann eigenvalue problems. Ann. Sc. Norm. Super. Pisa, Cl. Sci. 10, 729-755 (2011)

4. Miyajima, S, Motreanu, D, Tanaka, M: Multiple existence results of solutions for the Neumann problems via super- and sub-solutions. J. Funct. Anal. 262, 1921-1953 (2012)

5. Motreanu, D, Tanaka, M: Generalized eigenvalue problems of nonhomogeneous elliptic operators and their application. Pac. J. Math. 265(1), 151-184 (2013)

6. Kim, Y-H: A global bifurcation for nonlinear equations with nonhomogeneous part. Nonlinear Anal. 71, 738-743 (2009)

7. Ruiz, D: A priori estimates and existence of positive solutions for strongly nonlinear problems. J. Differ. Equ. 2004, 96-114 (2004)

8. Tanaka, M: Existence of the Fučík type spectrums for the generalized p-Laplace operators. Nonlinear Anal. 75, 3407-3435 (2012)

9. Faria, L, Miyagaki, O, Motreanu, D: Comparison and positive solutions for problems with (P,Q)-Laplacian and convection term. Proc. Edinb. Math. Soc. (to appear)

10. Zou, HH: A priori estimates and existence for quasi-linear elliptic equations. Calc. Var. 33, 417-437 (2008)

11. Lieberman, GM: Boundary regularity for solutions of degenerate elliptic equations. Nonlinear Anal. 12, $1203-1219$ (1988)

12. Fučík, S, John, O, Nečas, J: On the existence of Schauder bases in Sobolev spaces. Comment. Math. Univ. Carol. 13 163-175 (1972)

13. Deimling, K: Nonlinear Functional Analysis. Springer, New York (1985)

14. Anane, A: Etude des valeurs propres et de la résonnance pour l'opérateur p-laplacien. C. R. Math. Acad. Sci. Paris 305, 725-728 (1987)

15. Cuesta, M: Eigenvalue problems for the $p$-Laplacian with indefinite weights. Electron. J. Differ. Equ. 2001(33), 1-9 (2001)

doi:10.1186/1687-2770-2013-173

Cite this article as: Tanaka: Existence of a positive solution for quasilinear elliptic equations with nonlinearity

including the gradient. Boundary Value Problems 2013 2013:173.

\section{Submit your manuscript to a SpringerOpen ${ }^{\odot}$ journal and benefit from:}

- Convenient online submission

- Rigorous peer review

- Immediate publication on acceptance

- Open access: articles freely available online

- High visibility within the field

- Retaining the copyright to your article 